Research Paper

\title{
Blockade of Central GLP-1 Receptors Deteriorates the Improvement of Diabetes after lleal Transposition
}

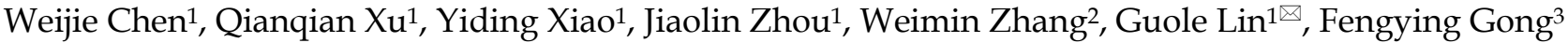 \\ 1. Department of Surgery, Peking Union Medical College Hospital, Chinese Academy of Medical Sciences, Shuaifuyuan 1\#, Beijing 100730, P. R. China. \\ 2. Clinical Laboratory of Peking Union Medical College Hospital, Chinese Academy of Medical Sciences, Shuaifuyuan 1\#, Beijing 100730, P. R. China. \\ 3. Department of Endocrinology, Key Laboratory of Endocrinology of the Ministry of Health, Peking Union Medical College Hospital, Chinese Academy of \\ Medical Sciences, Shuaifuyuan 1\#, Beijing 100730, P. R. China. \\ $\square$ Corresponding author: Guole Lin. Tel: 0086 13521624987. Fax: 0086010 69156002. Email: guolelin2002@163.com. Department of Surgery, Peking Union
} Medical College Hospital, Chinese Academy of Medical Sciences, Shuaifuyuan 1\#, Beijing 100730, P. R. China.

( ) Ivyspring International Publisher. Reproduction is permitted for personal, noncommercial use, provided that the article is in whole, unmodified, and properly cited. See http://ivyspring.com/terms for terms and conditions.

Received: 2016.08.21; Accepted: 2016.10.07; Published: 2016.11.23

\begin{abstract}
Background: The mechanism of improvement of type 2 diabetes mellitus induced by ileal transposition (IT) is undefined. Our aim was to investigate the possible role of central glucagon-like peptide 1 (GLP-1) after IT.

Methods: Ninety male diabetic rats were randomly divided into the IT, sham IT (S-IT) and control group. The food intake, glucose metabolism and GLP-1 level were measured. Subsequently, we administered GLP-1 antagonist via lateral brain ventricle cannula to block central GLP-1 receptor, and verified whether the food intake, glucose metabolism changed. And the activated pro-opiomelanocortin (POMC) neurons in different groups were compared after sacrifice.

Results: IT induced significant diabetic improvement with decreased maximum food intake and higher postprandial GLP-1 level. The GLP-1 level in cerebrospinal fluid increased in correlation with the plasma GLP-1 level. When the central GLP-1 receptor antagonist was given to the IT group rats, the improvement of the glucose level declined. The glucose level surged $(169.9 \pm 14.2)$ $\%$ during the oral glucose tolerance test, the range was larger than that before central blockade $((67.1 \pm 14.2) \%, P<0.001)$. Moreover, the POMC neuron number in the arcuate nucleus of the hypothalamus were reduced $(12.7 \pm 6.1$ at a magnification of $100 \times)$. The relative content level of POMC-derived peptides in the pituitary was lower $(0.1 \pm 0.05)$.

Conclusions: The central GLP-1 might play an important role in the remission of diabetes after IT. POMC neurons in the hypothalamus may be activated by the enhanced level of GLP-1 after IT.
\end{abstract}

Key words: type 2 diabetes mellitus, bariatric surgery, ileal transposition, glucagon-like peptide-1, neuron.

\section{Introduction}

Bariatric surgery induces profound and durable reduction of obesity and related metabolic conditions, such as type 2 diabetes mellitus (T2DM), even before significant weight loss [1]. As a bariatric surgery procedure, ileal transposition (IT) could also effectively improve T2DM with normal fasting blood glucose, insulin, and glycosylated hemoglobin level [2-3]. Common explanations for this response are based on the Hindgut Hypotheses [4]. Gastrointestinal changes by the IT make early nutrient contact with the terminal ileum. Accelerated delivery of unabsorbed nutrients lead to increasing stimulation of L-cells and secretion of glucagon-like peptide 1 (GLP-1).

Exaggerated GLP-1 secretion was speculated to be one of the most important factors for diabetes remission after bariatric surgery. It could stimulate insulin secretion, reduce food intake and improve insulin sensitivities in various aspects [5]. The 2016 ADA treatment guidelines even recommend GLP-1 receptor agonists in the treatment of poorly controlled T2DM [6]. Our previous study found that the level of 
GLP-1 in the cerebrospinal fluid (CSF) was significantly elevated after IT, and the pro-opiomelanocortin (POMC) neurons in arcuate nucleus were activated [7]. The POMC neuron is a specific population of arcuate nucleus neurons in the hypothalamus [8]. Theses neurons, known as "first order" neurons, project to several structures in the central nervous system, independently modulate autonomic nervous system output, affect different peripheral organs, and control energy balance [9].

Is the high level of central GLP-1 involved in the remission of T2DM after IT? Were those POMC neurons activated by the central GLP-1? There is no direct evidence from animal or human studies in the available literature. Our previous data did not prove the causation either. An understanding of the physiological mechanisms of the metabolic improvement is important for developing effective surgical techniques with fewer complications and anti-diabetic drugs without surgical risks. Therefore, the main aim of our study was to examine the effect of central GLP-1 and investigate the mechanism of remission of T2DM after IT.

\section{Materials and methods}

\section{Animals}

The experiments were carried out on ninety male Goto-Kakizaki (GK) T2DM rats (National Rodent Laboratory Animal Resources, Shanghai, China). GK rats are non-obese diabetic model that could reduce the impact of body weight change. And male rat model is easily to perform abdominal surgery. The GK rats were 10 weeks old with an initial body weight of 265-287 g. All rats were kept in individual cages under standard conditions (constant ambient temperature at $22{ }^{\circ} \mathrm{C}$ and humidity at $60 \%$ on a 12-hour light/dark cycle). They were fed with $5 \%$ fat rat chow diet and water ad libitum before the operation was performed. After 1-week acclimation, the rats were randomly assigned to three groups, each with thirty rats: the IT group, the sham IT (S-IT) group and the control group.

\section{Surgical procedures}

Under anaesthetization with $10 \%$ chloral hydrate solution, a stainless steel cannula was implanted into the lateral brain ventricle of all rats as described [10]. After 1-week of recovery, ileal transposition was performed in the IT group rat group [11]. A $10 \mathrm{~cm}$ ileal segment $5 \mathrm{~cm}$ proximal to the ileocecal valve was transected, transposed and anastomosed isoperistaltically with the jejunum $5 \mathrm{~cm}$ distal to the ligament of Treitz. The S-IT group rats received the sham surgery, which involved the same incision, transection and re-anastomosis of the gastrointestinal tract at multiple sites corresponding to the IT, except without ileum transposition. The control group rats did not received IT or sham surgery.

\section{Food intake and bodyweight}

The IT group rats were given non-residue diet (Ensure, Abbott, USA) for 2 days and fasting overnight pre-operatively (10 hours). Two hours after surgery, all rats had free access to water. Twenty-four hours after surgery, the non-residue diet was continued for 3 days, after which their normal diet (5\% fat rat chow diet) was not restricted. The S-IT group and the control group rats received the same amount of food ingested by the IT group rats. The aim of pair-fed was to control the body weight of the S-IT and control group, and reduce the potential impact to the glucose metabolism. The maximum 24-hour food intake was measured in three groups without food restriction after overnight fasting (10 hours). It is calculated by subtracting the weight of residue food from the weight of supply.

\section{Biochemical tests}

Blood glucose was measured using a glucometer (Roche One Touch $^{\circledR}$ Ultra, Lifescan, Johnson \& Johnson, Milpitas, USA) every week. Blood sample were collected from tail vein into chilled tubes containing a dipeptidyl peptidase IV inhibitor in EDTA solution postoperatively at the $2^{\text {nd }}, 6^{\text {th }}$ and $8^{\text {th }}$ week. After centrifugation $(1,000 \times g)$ at $4{ }^{\circ} \mathrm{C}$ for 15 minutes, plasma was immediately extracted and stored at $-80{ }^{\circ} \mathrm{C}$. The CSF $(10-20 \mu \mathrm{l}$ per rat) was collected from the lateral brain ventricle cannula and also stored at $-80{ }^{\circ} \mathrm{C}$. The postprandial GLP-1 in the plasma and CSF were collected at the first hour after non-restricted feeding, and were measured using an enzyme-linked immunosorbent assay (Uscn Life Science Inc., Wuhan, China).

\section{Oral glucose tolerance test}

Oral glucose tolerance test (OGTTs) were performed postoperatively at the $2^{\text {nd }}, 6^{\text {th }}$, and $8^{\text {th }}$ week [12]. The rats were fasting for 12 hours, a bolus of glucose $(2 \mathrm{~g} / \mathrm{kg}$ glucose) was given orally. Blood glucose was measured at the $15^{\text {th }}, 30^{\text {th }}, 60^{\text {th }}$, and $120^{\text {th }}$ minute.

\section{Intraperitoneal insulin tolerance test}

Intraperitoneal insulin tolerance tests (ITTs) were also performed postoperatively at the $2^{\text {nd }}, 6^{\text {th }}$, and $8^{\text {th }}$ week [12]. After fasting for 12 hours, $1 \mathrm{IU} / \mathrm{kg}$ insulin was intraperitoneally injected into each rat. Blood glucose was measured at the $30^{\text {th }}, 60^{\text {th }}, 90^{\text {th }}$, and $120^{\text {th }}$ minute. 


\section{Central GLP-1 receptor blockade}

At the $8^{\text {th }}$ week post-operation, all rats were fasting overnight. Each rat received an intracerebroventricular injection of $10 \mu \mathrm{l}$ exendin (9-39) (GLP-1 antagonist, Tocris, Bristol, UK) via 33-gauge injector and micro-syringe [13]. Subsequently, the OGTT was performed, and the 24-hour food intake was weighed. Four days later, all rats were fasting overnight and central GLP-1 receptors were again blocked, then the ITT was performed as previously described.

\section{Biochemical tests}

To verified the blockade effect of $10 \mu \mathrm{l}$ exendin (9-39), the IT group rats were assigned into two subgroups randomly at the $9^{\text {th }}$ week post-operation. A half of the IT group rats (IT-b rats) underwent an intracerebroventricular injection of $10 \mu \mathrm{l}$ exendin (9-39), while the other rats in the IT group had no injection. In the same way, a half of the S-IT group rats (S-IT-b rats) and half of the control group rats (control-b rats) accepted the intracerebroventricular injection of $10 \mu \mathrm{l}$ exendin (9-39). Subsequently, the cerebrospinal fluid, hypothalamus and pituitary tissues of all rats were collected in an anesthetized condition (a peritoneal injection of $10 \%$ chloral hydrate solution) [14-15]. The hypothalamic tissue specimens were fixed and stained by immunohistochemistry using rabbit anti-POMC precursor antibody (Abcam, Cambridge, UK) [16]. The number of POMC neurons was determined at 100× magnification [17]. The pituitary tissues were weighed and homogenized for extraction of POMC derivative. The amount of POMC derivative was analyzed by Western blot assay with an anti-adrenocorticotropic hormone antibody (Abcam, Cambridge, UK) [18]. The relative concentration of protein was quantified by densitometry using a Versa Doc 1000 Imaging System and Quantity One 4.4 software (Bio-Rad, Hercules, USA). All experiments were approved by the ethics committee of our hospital.

\section{Statistical analysis}

Data were reported as the mean \pm standard deviation. The statistic difference among three groups was analyzed using ANOVA, and the difference between two groups or two time-points was using Student's $t$-test (SPSS 19, SPSS, Inc, Chicago). A P value less than 0.05 was considered statistically significant. The area under the curve (AUC) for OGTT (AUC OGTT) and ITT $\left(\mathrm{AUC}_{\text {IтT }}\right)$ were calculated by trapezoidal integration.

\section{Results}

\section{Animal model}

There were no significant differences in the food intake $(P=0.16)$, body weight $(P=0.25)$ or fasting blood glucose $(P=0.09)$ among the groups before surgery. The average of maximum food intake was $17.3 \pm 1.5 \mathrm{~g}$, the body weight was $278.6 \pm 3.0 \mathrm{~g}$, and the fasting blood glucose was $12.1 \pm 1.8 \mathrm{mmol} / \mathrm{l}$. All operations were successfully performed (figure 1a). After surgery, two IT rats died due to intestinal obstruction on the $6^{\text {th }}$ and $14^{\text {th }}$ day after surgery, respectively. One rat in the S-IT group died from intraperitoneal infection 4 weeks after surgery. No other severe complication of surgery was observed.

\section{Food intake and weight}

The S-IT and the control group were pair-fed groups of the IT group, thus there was no statistic difference among three groups. When the food supply was not restricted, the maximum 24-hour food intake of the IT group was much less than that of the S-IT group or the control group. At the postoperative $8^{\text {th }}$ week, the maximum food intake of the IT group rats was $16.0 \pm 1.7 \mathrm{~g}$ and was significantly less than that of the S-IT group $(23.7 \pm 2.0 \mathrm{~g}, P<0.001)$ or that of the control group $(24.0 \pm 1.8 \mathrm{~g}, P<0.001)$.

GK rats are non-obese diabetic model, they did not gain much weight during the 9-week experiment period. At the postoperative $8^{\text {th }}$ week, the weight of the IT group rats was $281.1 \pm 13.0 \mathrm{~g}(P=0.09)$. Besides, there was no statistical difference among three groups. The S-IT group was $285.6 \pm 9.3 \mathrm{~g}$, and the control group was $285.8 \pm 4.9 \mathrm{~g}, P=0.14$ ).

\section{Glucose improvement}

The rats of the IT group showed amelioration of T2DM after IT surgery. The fasting blood glucose decreased from $12.1 \pm 1.9 \mathrm{mmol} / 1$ to $6.1 \pm 0.5 \mathrm{mmol} / 1$ ( 8 weeks after surgery, $P<0.001$ ), the AUC OGтT value decreased from $1949.4 \pm 179.0(\mathrm{mmol} / \mathrm{l}) \mathrm{min}$ to $999.1 \pm$ $52.6(\mathrm{mmol} / \mathrm{l}) \mathrm{min}(P<0.001)$, and the $\mathrm{AUC}_{\text {ITT }}$ value also decreased from $1236.6 \pm 150.2(\mathrm{mmol} / \mathrm{l}) \mathrm{min}$ to $615.0 \pm 43.3(\mathrm{mmol} / \mathrm{l}) \mathrm{min}(P<0.001)$. The curve of the fasting blood glucose, AUCOGTT and $\mathrm{AUC}_{\text {ITT }}$ are shown in figure 1.

There was no notable improvement of T2DM in the S-IT group or the control group rats. The fasting blood glucose, $\mathrm{AUC}_{\mathrm{OGTT}}$ and $\mathrm{AUC}_{\mathrm{ITT}}$ increased instead during the postoperative period. Consequently, the fasting blood glucose of the IT group rats $(6.1 \pm 0.5 \mathrm{mmol} / \mathrm{l})$ was remarkably lower than that of the S-IT group $(14.6 \pm 1.1 \mathrm{mmol} / \mathrm{l}, P<$ $0.001)$ or the control group $(14.5 \pm 0.9 \mathrm{mmol} / \mathrm{l}, P<$ $0.001)$ at the $8^{\text {th }}$ week post-surgery. The AUC 
value $(999.1 \pm 52.6(\mathrm{mmol} / \mathrm{l}) \mathrm{min})$ was also lower than that of the S-IT group rats $(2115.1 \pm 114.1$ (mmol/1)min, $P<0.001)$ and the control group (2167.9 \pm 159.3 (mmol/l)min, $P<0.001)$. In addition, the

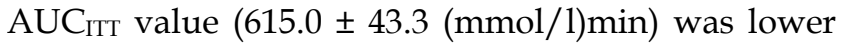
than that of the S-IT group rats $(1502.1 \pm 115.0$ $(\mathrm{mmol} / \mathrm{l}) \mathrm{min}, P<0.001)$ or the control group $(1528.6 \pm$ 82.8 (mmol/l)min, $P<0.001)$.

\section{GLP-1 level}

Higher postprandial GLP-1 levels were detected in plasma and CSF of the IT group rats. There were no significant differences in the GLP-1 level among the three groups before surgery. After surgery, the GLP-1 level in the IT group increase significantly, while the GLP-1 level in the S-IT group and the control group had no statistical change. At the $8^{\text {th }}$ week post-surgery, the level of plasma GLP-1 was $70.9 \pm$ $10.3 \mathrm{pmol} / 1$ in the IT group and was significantly higher than that of the S-IT group $(16.6 \pm 3.8 \mathrm{mmol} / \mathrm{l}$, $P<0.001)$ or the control group $(19.2 \pm 5.0 \mathrm{mmol} / \mathrm{l}, P<$ 0.001 , figure $2 \mathrm{a})$. In addition, the level of GLP-1 in the
CSF of the IT group was $32.4 \pm 4.7 \mathrm{pmol} / 1$ and was significantly higher than that of the S-IT group (7.4 \pm $1.9 \mathrm{mmol} / \mathrm{l}, P<0.001)$ or the control group $(5.2 \pm 1.8$ $\mathrm{mmol} / \mathrm{l}, P<0.001$, figure $2 \mathrm{~b}$ ). A linear regression relationship was observed between the CSF GLP-1 level and the plasma GLP-1 level. The CSF GLP-1 level increase significantly in correlation with the plasma GLP-1 level $(P<0.001)$. The Pearson Correlation coefficient was 0.982 .

\section{Central GLP-1 receptor blockade}

At the postoperative $8^{\text {th }}$ week, all rats received a dose of $10 \mu$ GLP-1 receptor antagonist via lateral brain ventricle cannula. Blockade of the central GLP-1 receptors worsen the improvement of type 2 diabetes mellitus in the IT group. The glucose level surged $(169.9 \pm 14.2) \%$ during the oral glucose tolerance test, the range was larger than that before central blockade $((67.1 \pm 14.2) \%, P<0.001)$. And blockade of central GLP-1 receptors did not affect the increase range during OGTT significantly in the other two groups (figure 2c).

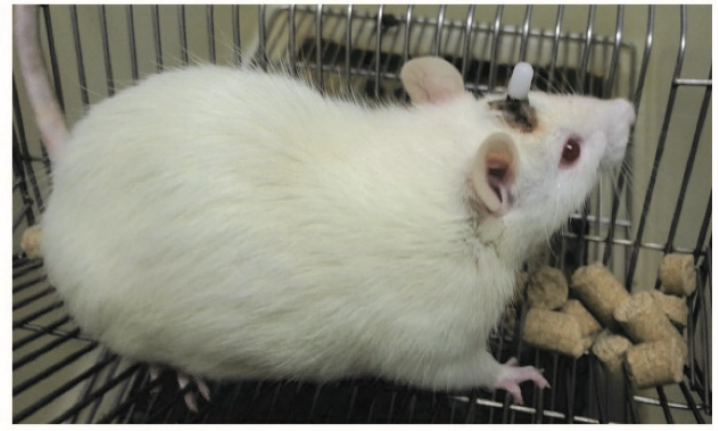

a

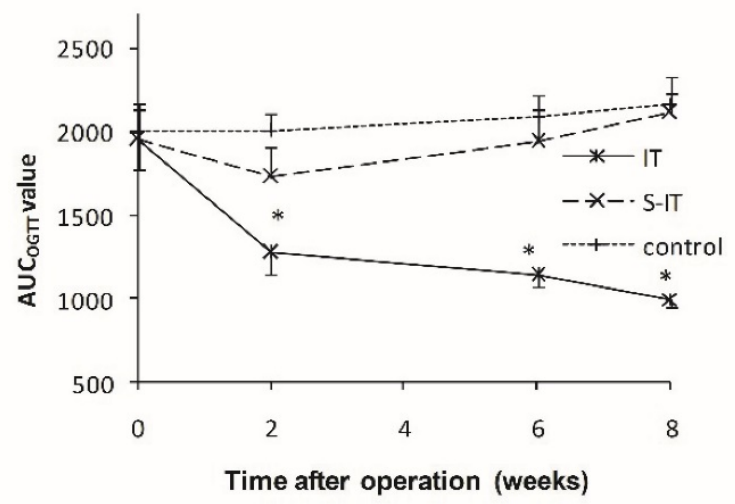

C

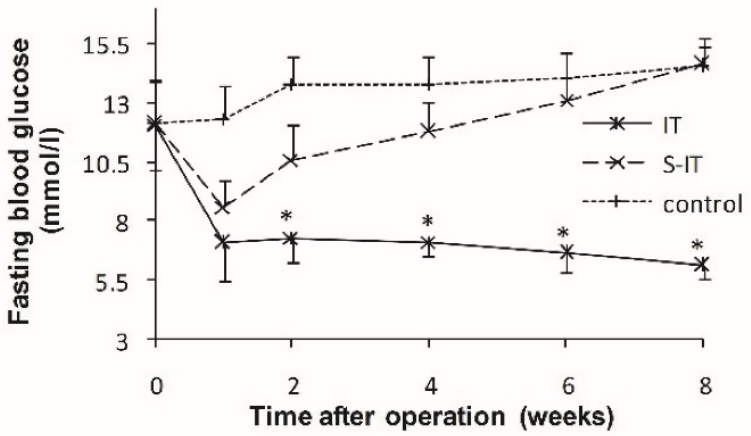

b

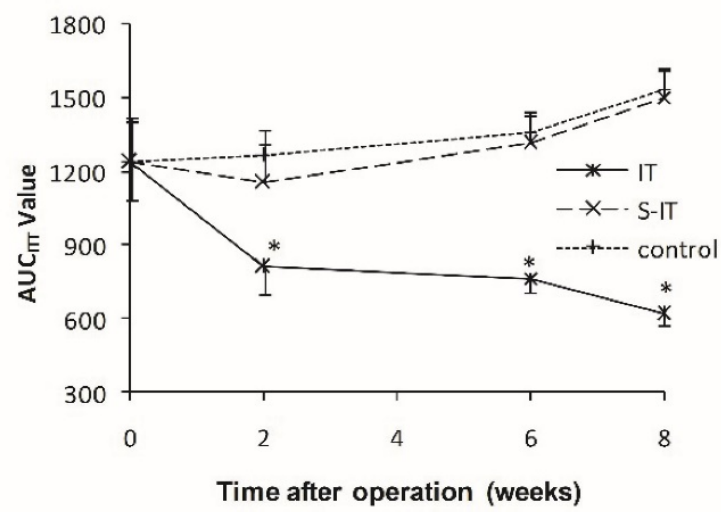

d

Figure 1 characteristics of the ileal transposition rat models. a, a non-obese GK rat model received lateral ventricle catheter and ileal transposition. b, the fasting glucose curve of three groups. The IT rats showed lower fasting glucose levels than the other two groups after surgery. c. the AUCOGTT during the postoperative period. d. the AUCITT during the postoperative period. The rats of the IT group showed amelioration of diabetes after surgery. The blood glucose level, AUCoGT and AUC ITT were less than that of the S-IT group. The asterisk means significantly different compared to the S-IT group $(P<0.05)$; AUC, areas under curve; GK rats, Goto-Kakizaki type 2 diabetic rats; IT, ileal transposition; ITT, insulin tolerance test; S-IT, sham ileal transposition. OGTT, oral glucose tolerance test. 


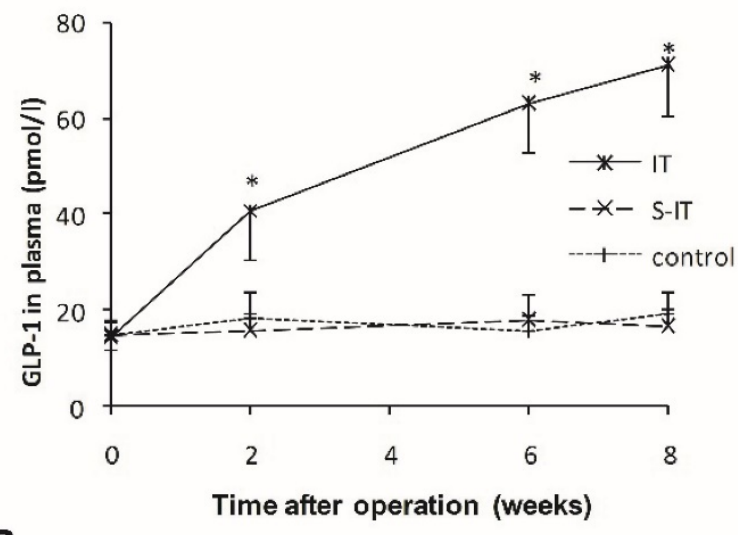

a

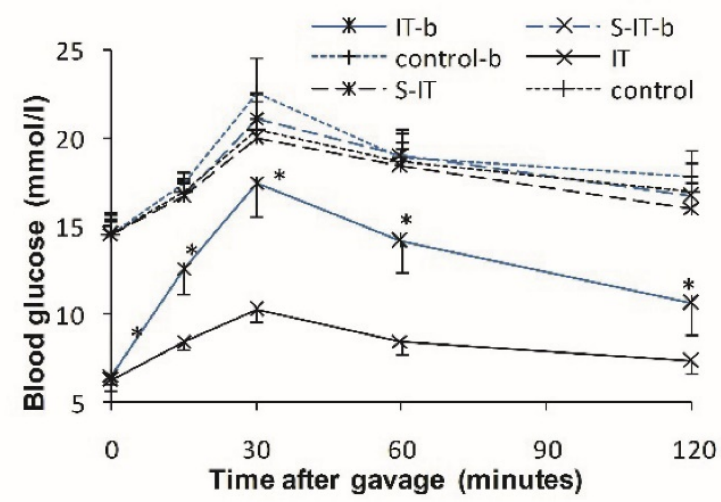

C

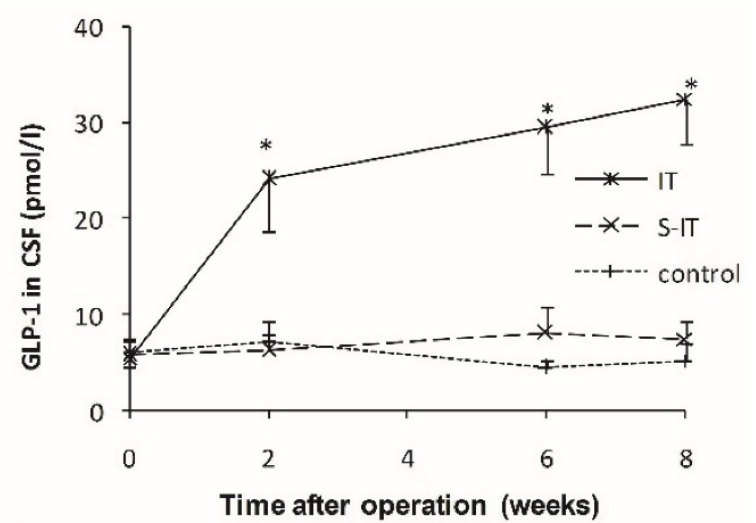

b

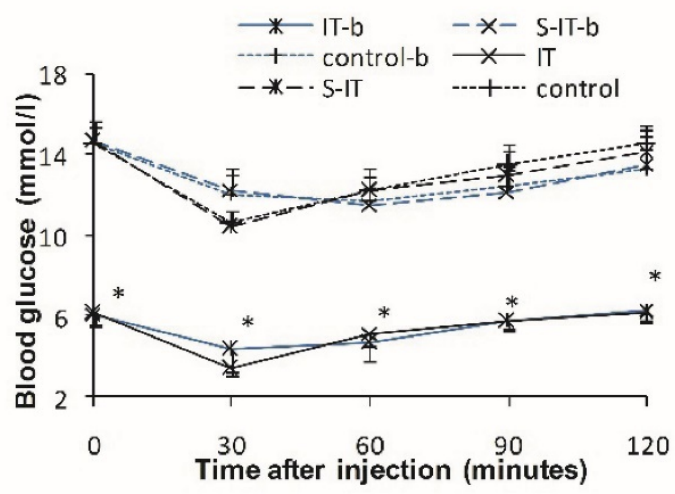

d

Figure 2 the effect of GLP-1 on the improvement of blood glucose in the IT group. a. the plasma GLP-1 level of three groups. b. the GLP-1 level in the CSF of three groups. The GLP-1 level of the IT rat group was higher than that of the S-IT group after surgery. c. the blood glucose level of OGTT before central GLP-1 receptor blockade at $6^{\text {th }}$ week post-operation (black lines) and after blockade at the $8^{\text {th }}$ week post-operation (blue lines). Blockade of central GLP-1 receptors reduced the improvement of diabetes in the IT group. $d$. the blood glucose level of ITT before and after central GLP-1 receptor blockade. The sensitivity of insulin did not deteriorate more than other two groups. The asterisk means significantly different compared to the S-IT group $(P<0.05)$; CSF, cerebrospinal fluid; control-b, control group rats accepted central GLP-1 receptor blockade; GLP-1, glucagon-like peptide 1; IT, ileal transposition; IT-b, ileal transposition rats accepted central GLP-1 receptor blockade; ITT, insulin tolerance test; OGTT, oral glucose tolerance test; S-IT, sham ileal transposition; S-IT-b, sham ileal transposition rats accepted central GLP-1 receptor blockade.

However, the relapse of the glucose deterioration in the IT group was partial. The glucose level of the IT group remained lower than that of the S-IT group or the control group. The peak glucose level of the IT group in the OGTT was $17.5 \pm 2.0$ $\mathrm{mmol} / 1$ after central blockade, still less than that of the S-IT group $(21.1 \pm 1.3 \mathrm{mmol} / \mathrm{l}, P<0.001)$.

Blockade of the central GLP-1 receptors worsen the insulin sensitivity of the IT group. When the ITT was performed in the IT group, the glucose level decreased (43.8 \pm 6.5) \% before blockade, and decreased $(30.1 \pm 12.3) \%$ after blockade $(P<0.001)$. The S-IT group and control group had a similar alter range of blood glucose after blockade (figure 2d).

The maximum 24-hour food intake increased significantly after central blockade. It rose from $15.4 \pm$ $1.1 \mathrm{~g}$ to $19.8 \pm 1.8 \mathrm{~g}(P<0.001)$, although it was still less than that of the S-IT group $(23.5 \pm 2.6 \mathrm{~g}, P<0.001)$ or the control group $(24.1 \pm 1.3 \mathrm{~g}, P<0.001)$. And the blockade did not affect the maximum food intake of the S-IT and control group. It was $21.8 \pm 3.2 \mathrm{~g}$ in the
S-IT group before blockade, and $24.8 \pm 3.3 \mathrm{~g}$ in the control group.

\section{Hypothalamic immunohistochemistry}

To verified the blockade effect of $10 \mu l$ exendin (9-39), the IT group rats were assigned into two subgroups randomly. The IT-b rats were given a GLP-1 antagonist before euthanasia, while the other rats in the IT group had no antagonist. The activated POMC neurons in the arcuate nucleus of hypothalamus were stained subsequently (figure 3a). The activated POMC neuron number of the IT-b rats was lower than that of the IT rats (non-blockade rats), although the GLP-1 level in the CSF did not fluctuate significantly. The mean neuron number at $100 \times$ magnification was $12.7 \pm 6.1$ in the IT-b rats, and $64.8 \pm$ 11.3 in the non-blockade IT rats $(P<0.001)$. Meanwhile, the S-IT-b rats and the control-b also accepted a GLP-1 antagonist before sacrifice. The mean neuron number was $9.6 \pm 4.7$ in the S-IT-b rats, $22.7 \pm 8.4$ in the S-IT rats, $10.2 \pm 6.6$ in the control-b 
rats, and $18.4 \pm 9.8$ in the control rats. The number of POMC neuron of IT group was more than that of the S-IT group $(P<0.001)$.

\section{The amount of POMC derivative in the pituitary}

The amount of POMC-derived peptides in the pituitary tissue was quantified by Western blot (figure $3 b)$. The relative content level of POMC-derived peptides of the IT-b rats $(0.1 \pm 0.05)$ was significantly lower than that of the non-blockade IT rats $(1.5 \pm 0.6, P$ $<0.001)$. Meanwhile, the POMC of the S-IT-b rats $(0.08$ $\pm 0.04)$ was less than that of the S-IT rats $(0.8 \pm 0.4)$, and POMC of the control-b $(0.07 \pm 0.04)$ rats was less than that of the control rats $(0.4 \pm 0.3)$. The POMC of the IT group was more than that of the S-IT group $(P<$ $0.001)$.

\section{Discussion}

Many animal and clinical studies have confirmed the fact that ileal transposition improves T2DM $[2,5,13]$. The results of our study are consistent with previous reports. After IT, fasting blood glucose decreased, the glucose tolerance and the insulin tolerance improved significantly. The mechanism of glucose improvement is complex and multifactorial. The generally accepted mechanism of IT is hypothesized to result from the enhanced secretion of GLP-1 from the terminal ileum stimulated by early arrival of food. The higher level of GLP-1 in the plasma was surely found in our study.

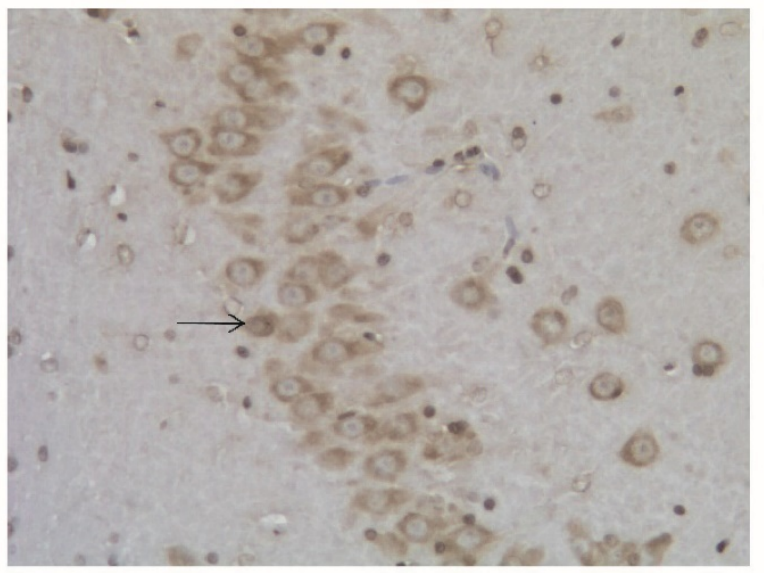

a

Figure 3 the blockade effect of exendin(9-39) in the IT group. a. the POMC neurons in the hypothalamic tissue at 100x magnification. The arrow indicates a POMC neuron stained by the immunohistochemistry stain. b, the amount of POMC derivative in the pituitary quantified by Western blot. Some IT rats (IT-b rats), S-IT rats (S-IT-b rats) and control rats (control-b rats) were given GLP-1 antagonist before sacrifice. The relative content level of POMC-derived peptides of the IT-b rats was significantly lower than that of the IT rats. The asterisk means significantly different compared to the S-IT group $(P<0.05)$; control-b, control group rats accepted GLP-1 receptor blockade; GLP-1, glucagon-like peptide 1; IT, ileal transposition; IT-b, ileal transposition rats accepted GLP-1 receptor blockade; POMC, pro-opiomelanocortin; S-IT, sham ileal transposition; S-IT-b, sham ileal transposition accepted GLP-1 receptor blockade.
What is noteworthy is that the higher level of GLP-1 in the CSF was found in the IT group rats. A linear regression relationship between the CSF GLP-1 level and the plasma GLP-1 level was observed. Besides, the POMC neurons and the POMC-derived peptides were more than that of the S-IT group rats. Considering that the GLP-1 receptor exists on the surface of hypothalamic POMC neurons, the central GLP-1 may affect the activities of hypothalamic POMC neurons [19]. We blocked the GLP-1 receptor of the IT rat group using central injection of exendin (9-39). The number of activated POMC neurons in the arcuate nucleus of the blockade rats less than that of the rats without blockade. In addition, the amount of the POMC derivative in the pituitary was less. It is reasonable to believe that the active POMC neurons were activated by central GLP-1.

The activated POMC neurons play an important role in the remission of diabetes after IT. The activated POMC neurons could decrease food intake and increase energy expenditure [9]. Ileal transposition has no resection or bypass of any small bowel or stomach. Additionally, there is no gastric restriction. However, ileal transposition decreased maximum food intake significantly compared to the S-IT or the control groups. The energy expenditure affected by POMC neurons included various aspects: adaptive thermogenesis, physical activity and basal metabolic rate [20]. POMC neurons provide dense synaptic inputs to pre-autonomic structures (such as paraventricular nucleus) and direct peripheral tissue activity (figure 4).
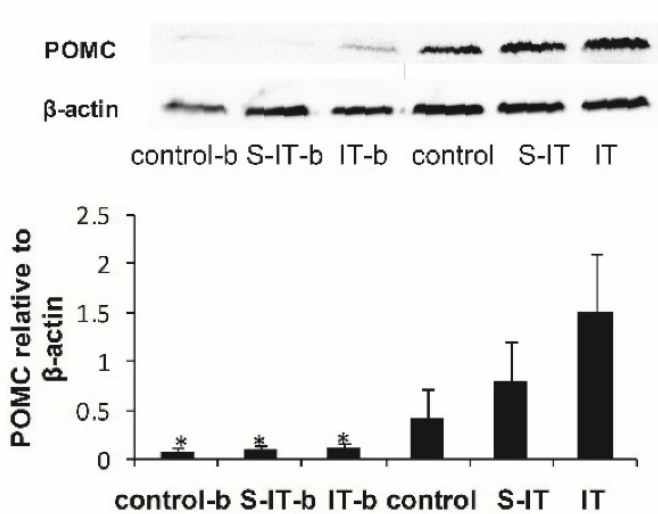

b 


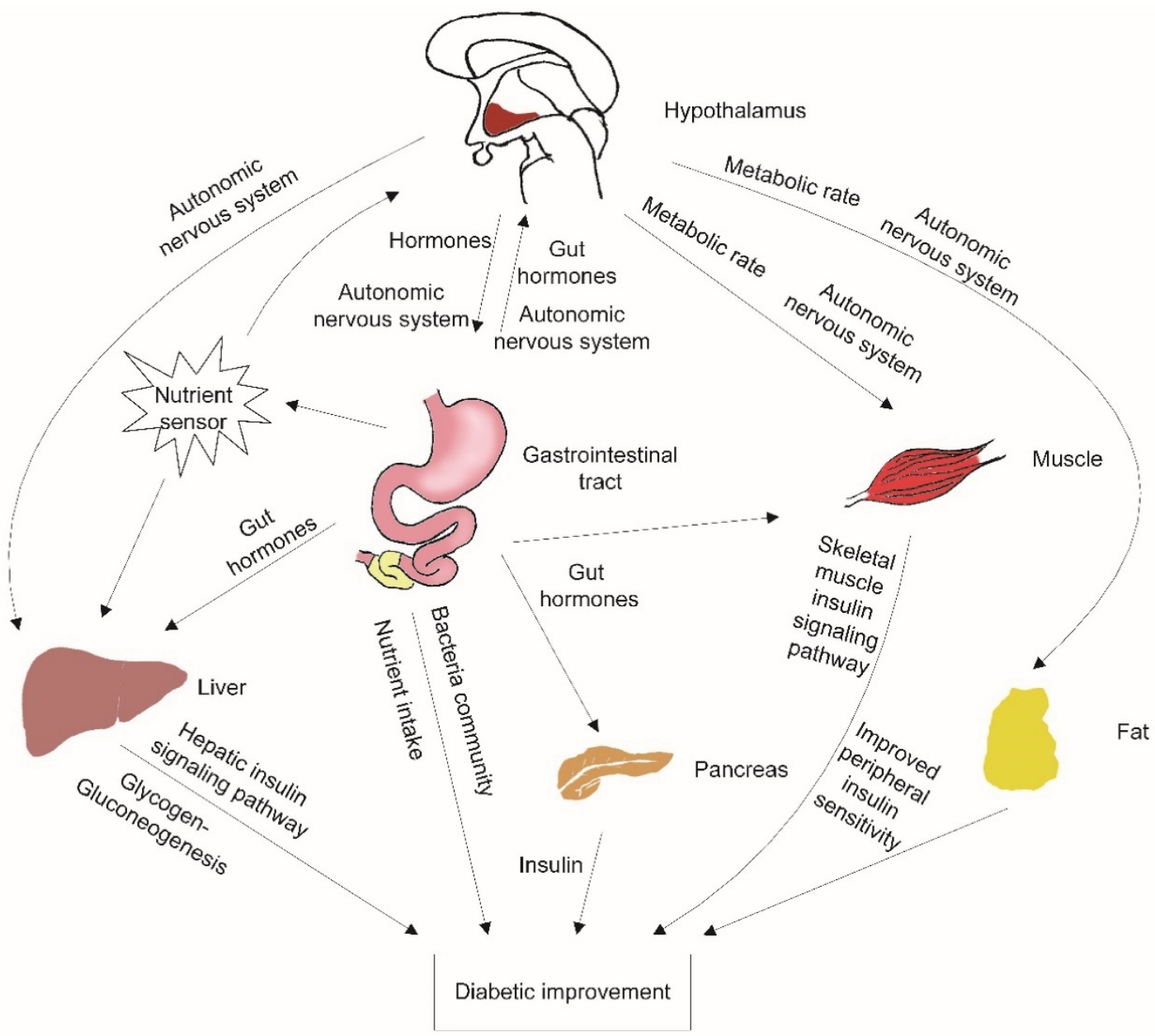

Figure 4 the possible mechanisms contributing to improved glucose metabolism after ileal transposition. The neurons in the hypothalamus could sense peripheral tissue activity, independently modulate autonomic nervous system output, affect different peripheral organs, and control energy balance. Nutrient malabsorption, gut hormone changes, peripheral insulin sensitivity improvement and changes in the gastrointestinal bacteria community are also the possible contributors to the amelioration of T2DM after surgery.

Ileal transposition offers a vivid example of how T2DM is improved immediately after surgery. As an obesity-related metabolic complication, T2DM involves the inappropriate conversion, storage and utilization of nutrients [9]. Re-homeostasis of energy by activated "energy neurons" may lead to the improvement of the glucose level of the IT rat group before the loss of body weight.

Therefore, blocking central GLP-1 receptors deteriorated these important improvements in the IT rats. Although the GLP-1 level in the CSF of the IT rat group did not fluctuate significantly, the level of glucose in the IT rat group increased significantly in OGTT, and the range was greater than it was before blockade was introduced. However, the relapse of the glucose deterioration was partial. This indicates that there are many other factors that ameliorate diabetes involved in the phenomenon. Nutrient malabsorption, gut hormone changes, peripheral insulin sensitivity improvement and changes in the gastrointestinal bacteria community have been proposed as possible contributors to the amelioration of T2DM after surgery (figure 4) [2-3, 21].

The arcuate nucleus of the hypothalamus contains several neuronal populations that are exquisitely integrated into the circulating signals of energy balance. In addition to the POMC neurons, the neuropeptide $\mathrm{Y}$ and the Agouti-related protein (NPY/AgRP) neurons are also involve in the autonomic nervous system mediated coordination of energy partitioning and peripheral organ activity. Our study is limited because we performed minimal investigations of other neurons. Despite the need for further studies, our results may provide new insight into the elucidation of the metabolic mechanisms of diabetes resolution after IT, which may have the potential to cure type 2 diabetes.

\section{Conclusion}

Ileal transposition improves glucose homeostasis in GK diabetes rat. The central GLP-1 might play an important role in the remission of diabetes after IT. In 
addition, POMC neurons in the hypothalamus may be activated by enhanced level of GLP-1 after IT.

\section{Abbreviations}

AUC: area under the curve; CSF: cerebrospinal fluid; GK: Goto-Kakizaki; GLP-1: glucagon-like peptide 1; IT: ileal transposition; IT-b: ileal transposition rats accepted central GLP-1 receptor blockade; ITT: insulin tolerance test; OGTT: Oral glucose tolerance test; POMC: pro-opiomelanocortin; S-IT: sham IT; S-IT-b: sham ileal transposition rats accepted central GLP-1 receptor blockade; T2DM: type 2 diabetes mellitus;

\section{Acknowledgements}

We thank American Journal Experts for the language polish.

\section{Competing Interests}

This work was supported by the National Natural Science Foundation of China (No.81400797/H0713). And we declare that we have no conflict of interest.

\section{References}

1. Schauer PR, Bhatt DL, Kirwan JP, Wolski K, Brethauer SA, Navaneethan SD, et al. Bariatric surgery versus intensive medical therapy for diabetes--3-year outcomes. The New England journal of medicine. 2014; 370: 2002-13.

2. Tomasz S, Dominika S, Iwona KS, Jodok F, Bronislawa SP, Marcin K, et al. Long-term Effect of Ileal Transposition on Adipokine Serum Level in Zucker (Orl)-Lepr(fa) Fatty Rats. Obes Surg. 2015; 25: 1848-57.

3. Oh TJ, Lee HJ, Cho YM. Ileal Transposition Decreases Plasma Lipopolysaccharide Levels in Association with Increased L Cell Secretion in Non-obese Non-diabetic Rats. Obes Surg. 2016; 26: 1287-95.

4. Reis CE, Alvarez-Leite JI, Bressan J, Alfenas RC. Role of bariatric-metabolic surgery in the treatment of obese type 2 diabetes with body mass index $<35$ kg/m2: a literature review. Diabetes Technol Ther. 2012; 14: 365-72.

5. Strader AD. Ileal transposition provides insight into the effectiveness of gastric bypass surgery. Physiol Behav. 2006; 88: 277-82.

6. Chamberlain JJ, Rhinehart AS, Shaefer CF, Jr., Neuman A. Diagnosis and Management of Diabetes: Synopsis of the 2016 American Diabetes Association Standards of Medical Care in Diabetes. Annals of internal medicine. 2016; 164: 542-52.

7. Chen W, Yan Z, Liu S, Zhang G, Sun D, Hu S. The changes of pro-opiomelanocortin neurons in type 2 diabetes mellitus rats after ileal transposition: the role of POMC neurons. J Gastrointest Surg. 2011; 15: 1618-24.

8. Solomou S, Korbonits $\mathrm{M}$. The role of ghrelin in weight-regulation disorders: implications in clinical practice. Hormones (Athens). 2014; 13: 458-75.

9. Joly-Amado A, Cansell C, Denis RG, Delbes AS, Castel J, Martinez S, et al. The hypothalamic arcuate nucleus and the control of peripheral substrates. Best Pract Res Clin Endocrinol Metab. 2014; 28: 725-37.

10. Bojanowska E, Stempniak B. Effects of tGLP-1 on feeding behaviour and neurohypophysial function under chronic osmotic stimulation. Physiol Behav. 2003; 78: 73-9.

11. Culnan DM, Albaugh V, Sun $\mathrm{M}$, Lynch $\mathrm{CJ}$, Lang $\mathrm{CH}$, Cooney RN. Ileal interposition improves glucose tolerance and insulin sensitivity in the obese Zucker rat. Am J Physiol Gastrointest Liver Physiol. 2010; 299: G751-60.

12. Burgos-Ramos E, Canelles S, Frago LM, Chowen JA, Arilla-Ferreiro E, Argente $\mathrm{J}$, et al. Improvement in glycemia after glucose or insulin overload in leptin-infused rats is associated with insulin-related activation of hepatic glucose metabolism. Nutr Metab (Lond). 2016; 13: 19.

13. Celik A, Cagiltay E, Ugale S, Asci M, Celik BO, Karaca C, et al. Diverted sleeve gastrectomy with ileal transposition in overweight, obese, and morbidly obese patients with type 2 diabetes: results of 1 -year follow-up. Surgery for obesity and related diseases : official journal of the American Society for Bariatric Surgery. 2016; 12: 541-9.

14. Shen L, Keenan MJ, Martin RJ, Tulley RT, Raggio AM, McCutcheon KL, et al. Dietary resistant starch increases hypothalamic POMC expression in rats. Obesity (Silver Spring). 2009; 17: 40-5.

15. Pritchard LE, Oliver RL, McLoughlin JD, Birtles S, Lawrence CB, Turnbull AV, et al. Proopiomelanocortin-derived peptides in rat cerebrospinal fluid and hypothalamic extracts: evidence that secretion is regulated with respect to energy balance. Endocrinology. 2003; 144: 760-6.

16. Choudhury AI, Heffron H, Smith MA, Al-Qassab H, Xu AW, Selman C, et al. The role of insulin receptor substrate 2 in hypothalamic and beta cell function. J Clin Invest. 2005; 115: 940-50.

17. Claret M, Smith MA, Batterham RL, Selman C, Choudhury AI, Fryer LG, et al. AMPK is essential for energy homeostasis regulation and glucose sensing by POMC and AgRP neurons. J Clin Invest. 2007; 117: 2325-36.

18. Slominski A, Zbytek B, Semak I, Sweatman T, Wortsman J. CRH stimulates POMC activity and corticosterone production in dermal fibroblasts. J Neuroimmunol. 2005; 162: 97-102.

19. Larsen PJ, Tang-Christensen M, Jessop DS. Central administration of glucagon-like peptide-1 activates hypothalamic neuroendocrine neurons in the rat. Endocrinology. 1997; 138: 4445-55.

20. Catarino T, Coletta M, LeGall J, Xavier AV. Kinetic study of the reduction mechanism for Desulfovibrio gigas cytochrome c3. European journal of biochemistry / FEBS. 1991; 202: 1107-13.

21. Seeley RJ, Chambers AP, Sandoval DA. The role of gut adaptation in the potent effects of multiple bariatric surgeries on obesity and diabetes. Cell metabolism. 2015; 21: 369-78. 